\title{
EMOCOLTURE: \\ INDAGINE RETROSPETTIVA IN UN REPARTO DI PEDIATRIA.
}

Tiddia F., Ferraguti P., Sanna M. P., Caboni M. G., Cocco P.L., Melis E., Pautasso M.

\section{Laboratorio di Analisi Chimico Cliniche e Microbiologia} Ospedale S. Giovanni di Dio, Cagliari.

Introduzione. Lo studio intende valutare la prevalenza, nel triennio luglio 2002 - giugno 2005, delle sepsi nei ricoverati nella Clinica Pediatrica del nostro Ospedale, e verificare eventuali variazioni significative nei tre periodi considerati. Metodi. Sono stati utilizzati flaconi pediatrici per aerobi ed anaerobi incubati per 7 giorni nel BacT Alert ${ }^{\circledR}$ 3DbioMerieux. Dei campioni segnalati come positivi sono stati allestiti: gram diretti, subcolture, identificazione biochimica e antibiogramma.

Risultati. Nel triennio considerato sono pervenute 568 emocolture, delle quali 36 sono risultate positive $(6 \%), 6$ nel primo anno (3\%), $16(8 \%)$ nel secondo e $14(7 \%)$ nel terzo. (Tab.1).

\begin{tabular}{lllccc}
\hline & & Pos. & $\%$ & g.+ & g.- \\
\hline Luglio $.02 \rightarrow$ Giugno .03 & 173 & 6 & 3 & 2 & 4 \\
\hline Luglio $.03 \rightarrow$ Giugno .04 & 192 & 16 & 8 & 12 & 4 \\
\hline Luglio $.04 \rightarrow$ Giugno .05 & 203 & 14 & 7 & 9 & 5 \\
\hline Totali & 568 & 36 & 6 & 23 & 13 \\
\hline
\end{tabular}

Sono stati isolati 13 gram negativi e 23 gram positivi.

Tra i gram positivi: $2 S$. aureo, $14 S$. coagulasi neg., 4 Str. Pneumoniae e 3 Streptococcus spp. Tra i gram negativi 6 K.E.S., 3 Salmonella spp e 4 Neisseriae meningitidis, quest'ultime nell'ultimo semestre dell'osservazione (gennaiogiugno 2005)

Conclusioni. L'analisi dei risultati consente di rilevare:

a) l'incremento temporale del numero di emocolture richieste (173 nel primo anno, 192 nel secondo, 203 nel terzo);

b) il numero di emocolture positive passa da 6 del primo anno a 16 nel secondo e 14 nel terzo;

c) una maggiore incidenza di gram positivi (23) rispetto ai negativi (13);

d) la presenza di tre Salmonelle;

e) l'isolamento, manifestatosi nell'ultimo semestre del-l'osservazione (gennaio-giugno 2005), di 4 Neisseriae meningitidis (gruppo B), che hanno causato quadri settici importanti, uno risoltosi con esito infausto.

Quest'ultimo aspetto assume rilevante importanza e di non facile spiegazione, trattandosi di un fenomeno epidemico, che seppur circoscritto, costituisce un segnale di allarme per il futuro. 